\title{
1-D Van der Waals Foams Heated by Ion Beam Energy Deposition
}

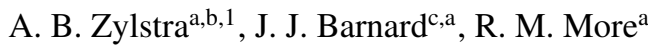 \\ ${ }^{a}$ Lawrence Berkeley National Laboratory, 1 Cyclotron Rd, Berkeley, CA 94720 USA \\ ${ }^{b}$ Pomona College Department of Physics, 610 N College Ave, Claremont, CA 91711 USA \\ ${ }^{c}$ Lawrence Livermore National Laboratory, 7000 East Avenue, Livermore, CA 94550 USA
}

\begin{abstract}
One dimensional simulations of various initial average density aluminum foams (modeled as slabs of solid metal separated by low density regions) heated by volumetric energy deposition are conducted with a Lagrangian hydrodynamics code using a van der Waals equation of state (EOS). The resulting behavior is studied to facilitate the design of future warm dense matter (WDM) experiments at LBNL. In the simulations the energy deposition ranges from 10 to $30 \mathrm{~kJ} / \mathrm{g}$ and from 0.075 to $4.0 \mathrm{~ns}$ total pulse length, resulting in temperatures from approximately 1 to $4 \mathrm{eV}$. We study peak pressures and temperatures in the foams, expansion velocity, and the phase evolution. Five relevant time scales in the problem are identified. Additionally, we present a method for characterizing the level of inhomogeneity in a foam target as it is heated and the time it takes for a foam to homogenize.
\end{abstract}

Key words: Warm dense matter, hydrodynamics

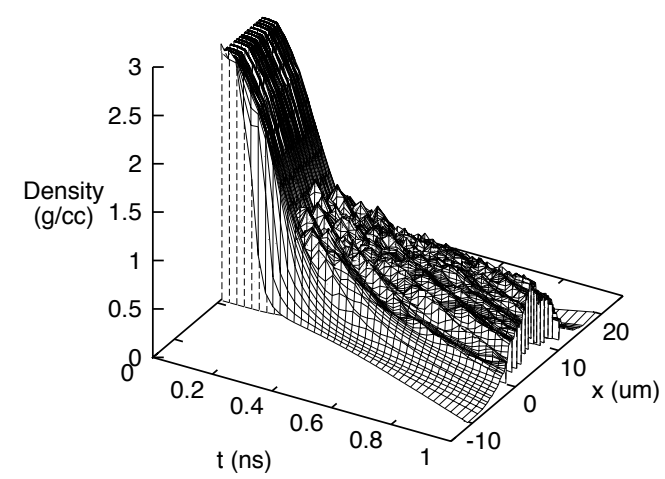

Figure 1: Density vs time and space, $30 \%$ liquid density $10 \mu \mathrm{m}$ thick foam (modeled as 15 slabs of solid material) heated by a $30 \mathrm{~kJ} / \mathrm{g} 0.5 \mathrm{~ns}$ pulse.

\section{Introduction}

Heavy ion accelerators have recently excited interest for near term warm dense matter (WDM) studies as well as long term potential applications to inertial fusion energy experiments $[1,11]$. For fundamental physics studies in the WDM regime heavy ion accelerators have the advantage that ion energies can be adjusted so that the beam deposition is nearly uniform throughout the target. The Neutralized Drift Compression Experiment (NDCX-I) $[9,8]$ at LBNL is carrying out experiments on metals in the two-phase regime [3]. A planned experiment NDCX-II, at LBNL, will explore higher temperatures [5], including temperatures above the critical point. NDCX-II will

\footnotetext{
${ }^{1}$ Corresponding author. Present address:

Massachusetts Institute of Technology, 77 Massachusetts Ave, NW17-256, Cambridge, MA 02139, USA. Tel.: +1-617-324-2322, fax: +1-617-258-7929, E-mail address: zylstra@mit.edu.
}

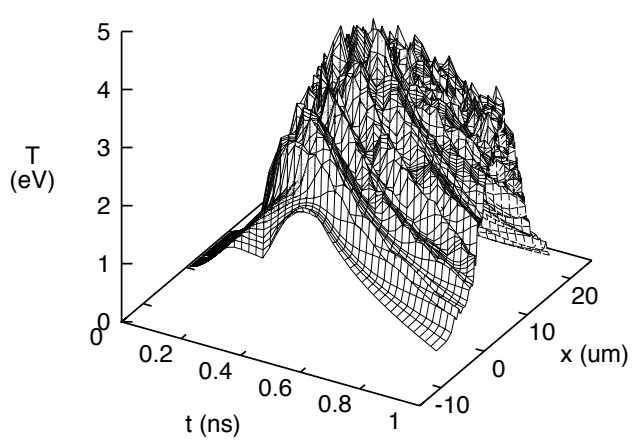

Figure 2: Temperature vs time and space, $30 \%$ liquid density $10 \mu \mathrm{m}$ thick foam heated by a $30 \mathrm{~kJ} / \mathrm{g} 0.5 \mathrm{~ns}$ pulse (same as in Figure 1).

run a $2.8 \mathrm{MeV} \mathrm{Li} \mathrm{beam} \mathrm{with} \mathrm{peak} \mathrm{central} \mathrm{fluence} 30 \mathrm{~J} / \mathrm{cm}^{2}$ corresponding to $20 \mathrm{~kJ} / \mathrm{g}$ in $\mathrm{Al}$ with a range of roughly $4 \mu \mathrm{m}$.

Previous simulation work on NDCX targets heated by heavy ion beams focused specifically on certain experimental configurations, including solid foils, with 1-D and 2-D simulation codes [1]. In this paper we focus on the simulation of metallic foams. Foam targets are of interest due to potential Inertial Confinement Fusion (ICF) uses [6] as well as for studying physics in the WDM regime. We present highly idealized studies of this problem and examine the fundamental processes of homogenization and expansion of the foams as heating parameters are varied.

The objective of this study is to determine the parametric dependence of peak temperatures, pressures, and expansion velocities on the beam energy, pulse duration, and foam density. Effects of foam dynamics on material phase in the expansion are explored, and we also determine the characteristic time scales for homogenization of the foam and the level of homogeneity expected. 


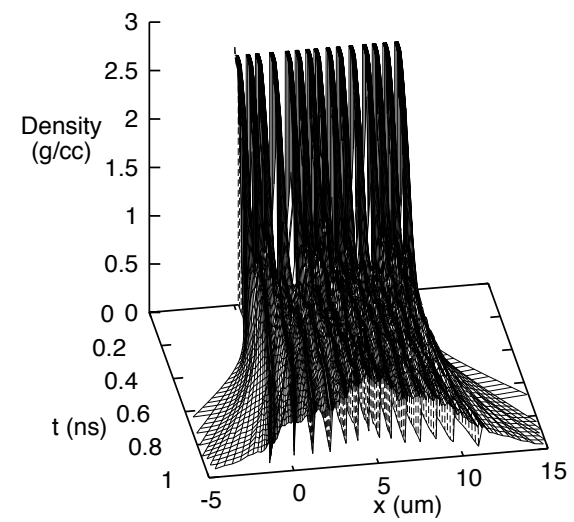

Figure 3: Density vs time and space, $30 \%$ liquid density $10 \mu \mathrm{m}$ thick foam heated by a $30 \mathrm{~kJ} / \mathrm{g} 0.5 \mathrm{~ns}$ pulse (rotated view of Figure 1 ).

Volumetrically heated foam targets have attracted recent interest. Tahir et al. have proposed using porous targets at GSI for critical point and two-phase studies, simulating potential targets without porous effects [10]. Efremov et al. present experimental and computational studies of pore collapse effects in a foam, similarly using a 1-D Lagrangian code but with a MieGruneisen equation of state [4]. Tauschwitz et al. use a 1-D Lagrangian Mie-Gruneisen code to study potential foam targets for GSI equation of state measurements in the WDM regime [12]. Their foam structure is highly similar to ours with a similar energy deposition in a much longer pulse length. Unlike these previous works this paper presents a fundamental study of the dynamics of foam targets over a large parameter space, and our focus is on heating times that are comparable to homogenization times.

\section{Methods}

For the simulations in this paper we use a one dimensional Lagrangian hydrodynamic code called DISH written by R. More [7]. Since the ion beam heating experiments we are modeling have target spot sizes much larger than the target depths a 1-D model is a good approximation in the center of the beam and computationally attractive. Higher dimensional foams may include more complex physics. We use a Van der Waals equation of state including the Maxwell construction, the simplest EOS that captures the phase transition between liquid and gas. The present DISH simulations are non-viscous and do not include ionization, heat conduction, or radiation transport, and are thus highly idealized.

The radiation energy density per gram is $4 \sigma T^{4} / \rho c$, which at a temperature of $4 \mathrm{eV}$ is $\sim 10^{-3} \mathrm{~J} / \mathrm{g}$, where $\sigma$ is the StephanBoltzmann constant, compared to the matter energy density per gram $\epsilon$ of $\sim 30 \mathrm{~kJ} / \mathrm{g}$. Similarly, the radiative energy flux is $\sigma T^{4} \sim 32 \mathrm{MW} / \mathrm{cm}^{2}$, whereas the hydrodynamic energy flux is $\sim \rho \epsilon c_{s} \sim 24 \mathrm{GW} / \mathrm{cm}^{2}$, suggesting that neglecting radiation effects should not significantly affect the dynamics.

Similarly, heat conduction should be relevant on a time scale $\tau_{\text {hc }} \sim\left(\rho C_{v} / \kappa\right) L^{2}$, where $C_{v}$ is the specific heat at constant volume $\sim 0.9 \mathrm{~J} / \mathrm{gK}, \kappa$ is the conductivity $\sim 2.4 \mathrm{~W} / \mathrm{cm} \mathrm{K}$ and $L$ is the characteristic thermal gradient length scale. So $\tau_{\mathrm{hc}} \sim\left(1 \mathrm{~s} / \mathrm{cm}^{2}\right)(\mathrm{L} / 1 \mathrm{~cm})^{2}$. For characteristic length scales of 10 $\mu \mathrm{m}$, the timescale for heat conduction would be $1 \mu \mathrm{s}$, which is much longer than the simulation times. However for a single slab of thickness $0.067 \mu \mathrm{m}$ (for a $10 \%$ foam) the heat conduction time scale is $4 \times 10^{-11} \mathrm{~s}$, whereas the hydrodynamic time scale for the same slab is $L / c_{s} \sim 2 \times 10^{-11} \mathrm{~s}$. In this case heat conduction over individual slabs is not completely ignorable. Thus, our neglect of heat conduction should not effect the macro-scale dynamics, but temperature spikes observed during slab collisions in the simulations probably overestimate the temperature, since heat conduction would have the effect of reducing the extremes in temperature.

Since the Van der Waal equation of state does not specify the electron number density, it does not include any effects of ionization. At $1-2 \mathrm{eV}$ ionization is relatively weak for atomic Al. However, in the liquid state, conduction electrons act in some ways like free electrons. Nevertheless, the critical point and the liquid-vapor boundary of the Van der Waal fluid is chosen to be in the same regime as expected for $\mathrm{Al}$, so qualitative features of the hydrodynamics in this study are expected to be present when a more detailed EOS is used for the simulation.

Simulations are driven by initial and boundary conditions as well as energy deposition in the form of a spatially uniform pulse with a parabolic time dependence. Unless otherwise specified, we use aluminum for the foams and foils. The density (Figures 1,3) and temperature (Figure 2) are plotted versus time and space for a typical foam.

We assume that the transition between solid and liquid is rapid, and therefore focus on the phase transition between liquid and vapor. Liquid foils are initialized as a continuous set of Lagrangian cells, created with a tapering routine such that the cells near the edge are smaller in size than the central cells, allowing for better expansion accuracy. Foams are initialized as a series of slabs of liquid material separated by gaps filled with a very low density gas. The nominal construction used was fifteen liquid slabs of material modeled with 315 Lagrangian cells, which is considered to be the minimum number of slabs and cells per slab to describe the essential foam physics. Gap sizes were slightly randomized, and the same randomization was used for all standard foam runs. Here the size for the $i$ th gap is given by

$$
d_{\text {gap }, \mathrm{i}}=\bar{d}_{\text {gap }} \times \delta_{\text {gap }, \mathrm{i}}
$$

where $\delta_{\text {gap }}=1 \pm \epsilon$ where $\epsilon$ is a random number of magnitude $|\epsilon| \leq 0.7$.

Changing the randomization was found to affect macro parameters by only $2-3 \%$. Foam average density was adjusted by changing the relative thickness of the slabs and gaps. Our standard foams and foils were $10 \mu \mathrm{m}$ in total thickness.

For the results in sections four through six we varied the average foam density from $15 \%$ to fully liquid foils. This was achieved by scaling the liquid slab sizes and inversely scaling the gaps, while holding the total foam thickness constant. Even though average foam densities as low as $1 \%$ are constructed in the lab, $10-15 \%$ minimum density is more common, especially 
for target physics. The foam density is defined as

$$
\rho_{\text {foam }}=\frac{\rho_{\text {liquid }}}{1+d_{\text {gap }} / d_{\text {liquid }}},
$$

where $d_{\text {gap }}$ and $d_{\text {liquid }}$ are the average thickness of the slabs of liquid material in the initial foam and the gas-filled gaps between them. We normally quote fractional liquid densities:

$$
\left(\rho_{\text {foam }} / \rho_{\text {liquid }}\right)=\frac{1}{1+d_{\text {gap }} / d_{\text {liquid }}} .
$$

The pulse length was varied from $0.075 \mathrm{~ns}$ to $4.0 \mathrm{~ns}$, and the energy deposition varied from $10 \mathrm{~kJ} / \mathrm{g}$ to $30 \mathrm{~kJ} / \mathrm{g}$. This spans parameter ranges of interest for upcoming ion beam experiments at NDCX II which will be $\sim 20 \mathrm{~kJ} / \mathrm{g}$ deposition in $\sim 1 \mathrm{~ns}$.

Peak parameters, i.e. the temperature or pressure of the foam, were calculated as a mass weighted mean of the center third of the Lagrangian cells. For calculating the homogenization of a foam we use three methods: first, a volume weighted average density to calculate the rms deviance of the density from the mean, normalized to the mean, over the center third of the Lagrangian cells. This gives us a measure that is high when the foam has large inhomogeneities, such as when it is initially partitioned into liquid slabs, and low when the foam slabs have collided and homogenized. We calculate a homogenization time from this measure by finding the mean and standard deviation of several time points at the end of the run, and then defining the homogenization time as the time at which the homogenization level is within two standard deviations of the final value.

In simulations where the homogenization time is small compared to the total length of the simulation a linear trend in this value of the homogenization is observed at the tail of the experiment due to macro-rarefaction waves, where here the rarefaction wave resulting from the expansion of the bulk material is called the macro-rarefaction. To account for this a second method of calculating the homogenization uses the result of the first and subtracts this linear trend, recalculating the homogenization time. This method is only used when the macrorarefaction affects the homogenization calculations.

The code hydrodynamics was verified against an analytic solution in an initialized temperature slab expansion, i.e. a double rarefaction, and agreed to better than $0.5 \%$. Convergence was tested for typical cases by decreasing the time step by an order of magnitude, resulting in no measurable change in macro parameters, and by increasing the number of Lagrangian zones by an order of magnitude, which results in a change of macro parameters $\sim 4-8 \%$ for typical cases.

\section{Time Scales in the Problem}

\subsection{Sound Speed}

Relevant time scales in the problem will be inherently dependent on the Van der Waals sound speed. Here,

$$
\left.c_{s}^{2} \equiv \frac{\partial p}{\partial \rho}\right|_{s}=\left.\frac{\partial p}{\partial \rho}\right|_{T}-\left.\frac{\partial p}{\partial T}\right|_{\rho}\left(\frac{\left.\frac{\partial s}{\partial \rho}\right|_{T}}{\left.\frac{\partial s}{\partial T}\right|_{\rho}}\right),
$$

where for a Van der Waals fluid

$$
\begin{aligned}
& p=\frac{\rho k T}{m_{a}\left(1-b_{v d w} \rho\right)}-a_{v d w} \rho^{2}, \\
& s=\frac{k}{m_{a}} \ln \left(m_{a} \frac{1-b_{v d w} \rho}{\rho \lambda^{3}}\right), \lambda=\sqrt{\frac{h^{2}}{2 \pi m_{a} k T}} \\
& \epsilon=\frac{3 k T}{2 m_{a}}-a_{v d w} \rho,
\end{aligned}
$$

where $m_{a}$ is the atomic mass. So we have, in the Van der Waals EOS,

$$
\begin{aligned}
\frac{\partial p}{\partial \rho} & =\frac{k T}{m_{\mathrm{a}}} \frac{1}{\left(1-b_{v d w} \rho\right)^{2}}-2 a_{v d w} \rho, \\
\frac{\partial p}{\partial T} & =\frac{k \rho}{m_{\mathrm{a}}\left(1-b_{v d w} \rho\right)}, \\
\frac{\partial s}{\partial T} & =\frac{3}{2} \frac{k}{m_{\mathrm{a}} T}, \\
\frac{\partial s}{\partial \rho} & =-\frac{k}{m_{\mathrm{a}}\left(1-b_{v d w} \rho\right) \rho} .
\end{aligned}
$$

$a_{v d w}$ and $b_{v d w}$ are the Van der Waals coefficients:

$$
\begin{aligned}
a_{v d w} & =\frac{27 b_{v d w} T_{c r i t}}{8 c_{v d w}}, \\
b_{v d w} & =\frac{1+\sqrt{1-(32 / 27)\left(T_{r e f} / T_{c r i t}\right)}}{2 \rho_{\text {solid }}},
\end{aligned}
$$

Since $T_{\text {ref }} \approx 1 / 40 \mathrm{eV}$ and $T_{\text {crit }} \approx 1 \mathrm{eV}$, it is a good approximation to let $b_{v d w}=1 / \rho_{\text {solid }}$.

Substituting these expressions into Equation 4 gives us that

$$
c_{s}^{2}=\frac{5}{3} \frac{T}{c_{v d w}} \frac{1}{\left(1-b_{v d w} \rho\right)^{2}}-2 a_{v d w} \rho .
$$

This is plotted versus $T$ and $\rho$ in Figure 4. These formulas apply in the one-phase part of the $\rho-T$ space. In the two-phase region, the sound speed is obtained from values on the phase boundary by the Maxwell construction [2].

The speed of sound as the foams are heated will thus depend on the time evolution of the temperature and density. We can estimate the time dependence of the temperature by taking the beam power per unit of mass,

$$
P=\frac{3}{2} \frac{E_{\text {dep }}}{t_{\text {pulse }}}\left(1-\frac{4\left(t-t_{\text {pulse }} / 2\right)^{2}}{t_{\text {pulse }}^{2}}\right), \quad \frac{-t_{\text {pulse }}}{2} \leq t \leq \frac{t_{\text {pulse }}}{2}
$$

where $E_{\mathrm{dep}}$ is the total energy deposition per unit mass and $t_{\text {pulse }}$ is the pulse length, and integrating over time while dividing by the specific heat:

$$
\begin{aligned}
& T \approx \int \frac{P d t}{\left.\frac{d E}{d T}\right|_{\rho}},\left.\quad \frac{d E}{d T}\right|_{\rho}=\frac{3 k}{2 m_{a}} \\
& T \approx \frac{E_{\mathrm{dep}} m_{a}}{k}\left[\left(\frac{t}{t_{\mathrm{pulse}}}\right)^{2}-\frac{1}{3}\left(\frac{t}{t_{\mathrm{pulse}}}\right)^{3}\right]+T_{0} .
\end{aligned}
$$




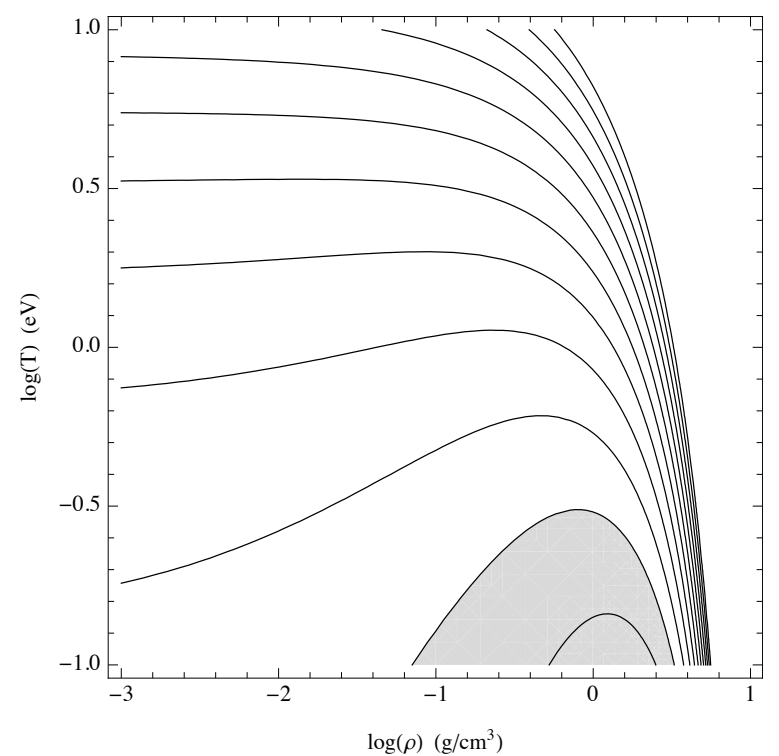

Figure 4: Contour plot of $c_{s}^{2}$. Contours are spaced at $2.5 \mathrm{~km} / \mathrm{s}$ increments. The shaded region corresponds to where $c_{s}^{2}<0$ in the Van der Waals sound speed without Maxwell construction.

\subsection{Definitions}

We have identified five relevant time scales in the problem: $t_{\text {micro }}, t_{\text {collision }}, t_{\text {homog }}, t_{\text {macro }}$, and $t_{\text {pulse. }}$. The micro-rarefaction time, or $t_{\text {micro }}$, is the time at which rarefaction waves in the slabs of the foam propagate to the centers of the slabs. The slab collision time, or $\mathrm{t}_{\text {collision, }}$, is the time at which the slabs collide. The homogenization time, or $t_{\text {homog }}$, is the time at which the foam has homogenized. The macro-rarefaction time, $t_{\text {macro }}$, is simply when the rarefaction waves in the bulk material have propagated to the center of the foam. Finally, $t_{\text {pulse }}$ is the end of the pulse.

In the parameter space examined in this study $t_{\text {micro }}$ is observed to always be the shortest of the time scales. In a shorter pulse regime this timescale would be more interesting.

We can estimate some of the time scales. The micro rarefaction time is of course the time necessary for a rarefaction wave to propagate to the middle of a single liquid slab:

$$
\frac{d_{\text {liquid }}}{2}=\int_{0}^{t_{\text {micro }}} c_{s}(t) d t
$$

Since $t_{\text {pulse }}>>t_{\text {micro }}$ we observe that the temperature, Equation 17 , is approximately constant over this time scale.

We can use a perfect gas approximation to estimate the time for the rarefaction wave to propagate to the center of the slabs by:

$$
\begin{gathered}
\frac{d_{\text {liquid }}}{2} \approx c_{s 0} \frac{t_{\text {micro }}^{2}}{t_{\text {pulse }}}, \\
t_{\text {micro }} \approx \sqrt{\frac{d_{\text {liquid }} t_{\text {pulse }}}{2 c_{s 0}}} .
\end{gathered}
$$

Here $c_{s 0}$ is the sound speed at the end of the pulse, assuming no hydro motion. If the time for the slabs to collide is also small compared to the energy deposition timescale $\left(t_{\text {pulse }}\right)$ we can extend this:

$$
t_{\text {collision }} \approx \frac{t_{\text {micro }}}{3} \frac{d_{\text {gap }}}{d_{\text {liquid }}},
$$

where in an ideal gas EOS approximation the micro-rarefaction waves have an expansion rate of $3 c_{s}$.

We can also make some estimates of the macroscopic rarefaction time, depending on how $t_{\text {macro }}$ compares to $t_{\text {pulse }}$ :

$$
t_{\text {macro }} \approx \frac{d_{\text {macro }}}{2 \overline{c_{s}}} \quad\left(t_{\text {macro }}>>t_{\text {pulse }}\right),
$$

where $d_{\text {macro }}$ is the total width of the foam. In the other limit:

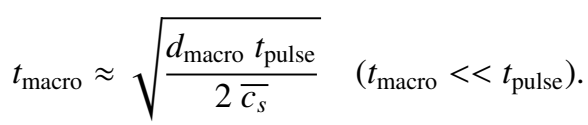

Here $\overline{c_{s}}$ is the sound speed of the homogenized foam as a Van der Waals fluid at the average density and temperature of the foam. When $t_{\text {pulse }} \sim t_{\text {macro }}$ such analytic approximations become complicated, since energy deposition occurs over several of these time scales relevant to stages of foam expansion and homogenization, and the continued heating affects the physics.

These simplified, analytic time scales are presented for order of magnitude estimates. The subsequent results for time scale comparisons are numeric.

\section{Comparing Foams to Foils}

\subsection{Peak Temperature}

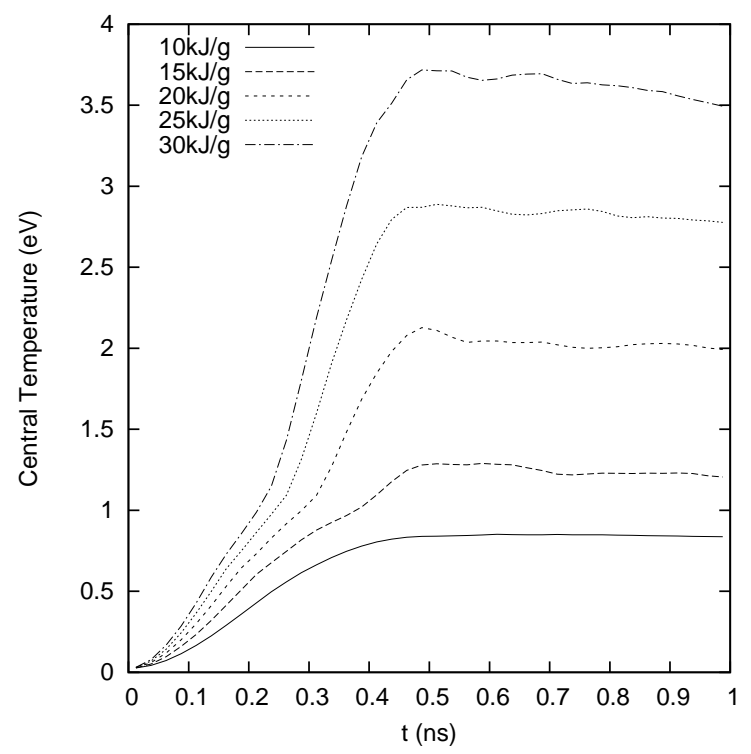

Figure 5: Central temperature vs time, $30 \%$ liquid density foam heated by a 0.5 ns pulse with various energy depositions.

In Figure 5 we show plots of the central temperature in $30 \%$ initial liquid density foams heated by a $0.5 \mathrm{~ns}$ pulse for five 
different energy depositions. In an idealized model, peak temperatures will occur at the end of the pulse, when all of the pulse energy has been deposited but before the foam expands. In reality a macro rarefaction wave propagates into the foam during energy deposition and can cool the center during energy deposition. Thus, if the macro rarefaction reaches the center before the end of the pulse the peak central temperature can occur before all energy deposition has occurred.

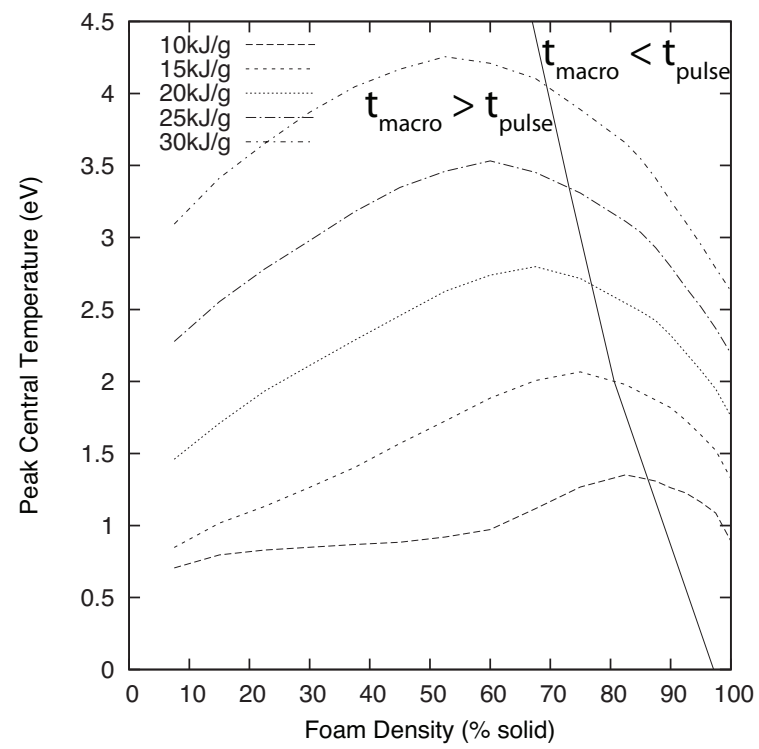

Figure 6: Peak central temperature observed vs initial foam density, $0.5 \mathrm{~ns}$ pulses. The solid line connects the points for each energy deposition trend for which $t_{\text {macro }}=t_{\text {pulse }}$. For all points $t_{\text {macro }}>t_{\text {collision }}, t_{\text {micro }}$.

We show the peak temperature vs the initial density of the foam in Figure 6, for $0.5 \mathrm{~ns}$ pulses of various energy depositions. The solid line denotes where $t_{\text {pulse }}=t_{\text {macro }}$. For higher densities, to the right of the solid line, we find that increasing the density increases the sound speed (see Figure 4) and thus decreases $t_{\text {macro }}$, which reduces the amount of energy deposited by the time the macro rarefaction wave propagates to the center of the foam $\left(t_{\text {macro }}<t_{\text {pulse }}\right)$, which in turn reduces the maximum temperatures reached. For lower densities, to the left of the solid line, we observe a slight increase to a peak temperature and then a long tail to lower temperatures. In these cases $t_{\text {macro }}>t_{\text {pulse }}$, so the macro expansion of the foam does not alter the temperature in the center by the end of the pulse - when the peak temperature occurs in these cases. We use a large section of the foam, equal to one third of the volume, as the defined center. As a result, the macro-rarefaction wave does have a small effect on the central temperature when $t_{\text {pulse }}$ is only slightly less than $t_{\text {macro. }}$. This accounts for the shift between the observed peaks in Figure 6.

For the low density tail of the curves we discover that as the slabs of liquid material in the foam expand into the gaps of the foam the material cools, and this is sufficient cooling to account for the trend in Figure 4.

We also observe a third effect during the foam heating process. When the slabs of liquid material expand into the gaps they collide with adjacent slabs. This compression can create

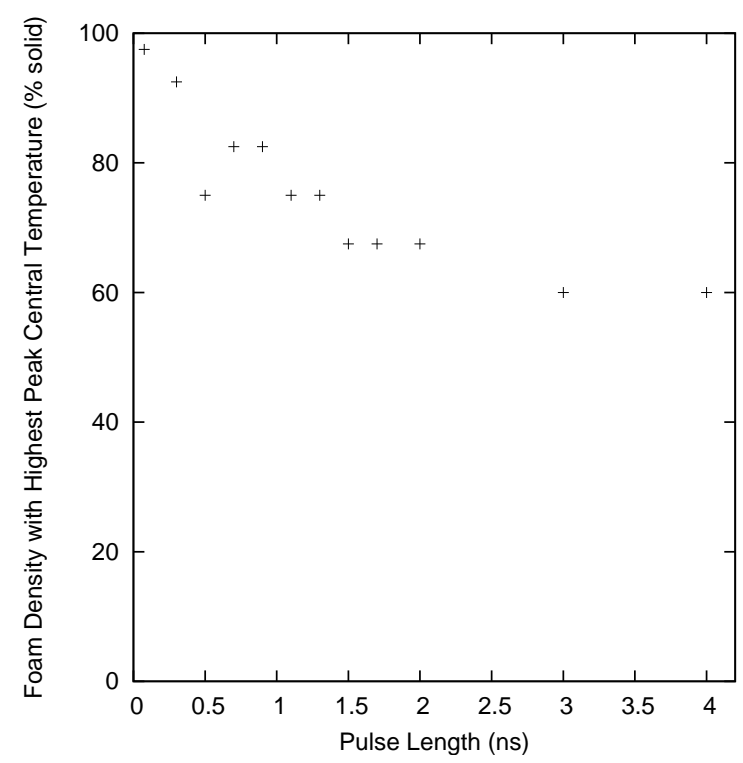

Figure 7: The foam density with the highest peak temperature vs pulse length, $15 \mathrm{~kJ} / \mathrm{g}$ energy deposition.

a momentary jump in the observed temperature. This effect is more prominent in lower density foams, since we use the same number of slabs for each foam a slab in a lower density foam has more space to accelerate before colliding with another slab. In special cases where $t_{\text {collision }}$ is comparable to $t_{\text {pulse }}$ this effect can create maximum central temperatures.

In Figure 7 we plot the densities at which the peak temperature occurs for various pulse lengths, all with one value for the energy deposition. We see that at lower pulse lengths the peak temperature occurs in a higher density foam. This is because $t_{\text {macro }}$ decreases as the density increases, and so for lower $t_{\text {pulse }}$ we have that $t_{\text {pulse }}=t_{\text {macro }}$ at a higher density. Likewise, longer pulses experience a peak temperature for lower densities.

\subsection{Peak Pressure}

We similarly calculate the peak pressure observed in the foam center and examine the parameter dependence. Figures 8 and 9 show this for varying energy depositions in $0.5 \mathrm{~ns}$ and $1.5 \mathrm{~ns}$ pulses. The solid lines connect the points on each constant energy deposition trend for which $t_{\text {pulse }}=t_{\text {macro }}$. At higher foam densities in Figures 8 and 9 we can see sharp increases in the peak pressure. At higher densities the peak pressure measured occurs before the initial slabs of the foam expand and homogenize. Since the densities are close to $\rho_{\text {liquid }}$ at these conditions the Van der Waals equation of state pressure (given in Eq. 5) diverges since $b_{v d w} \approx 1 / \rho_{\text {liquid. }}$.

Unlike in the previous section we see a maximum in this parameter, i.e. the pressure, at densities which do not correspond to $t_{\text {pulse }}=t_{\text {macro }}$. This is especially clear in the longer pulse case, Figure 9. We thus observe that maximizing the pressure for given pulse parameters in this regime requires a higher density foam than if the temperature is maximized. This is entirely the result of the density dependence of the Van der Waals pressure. 


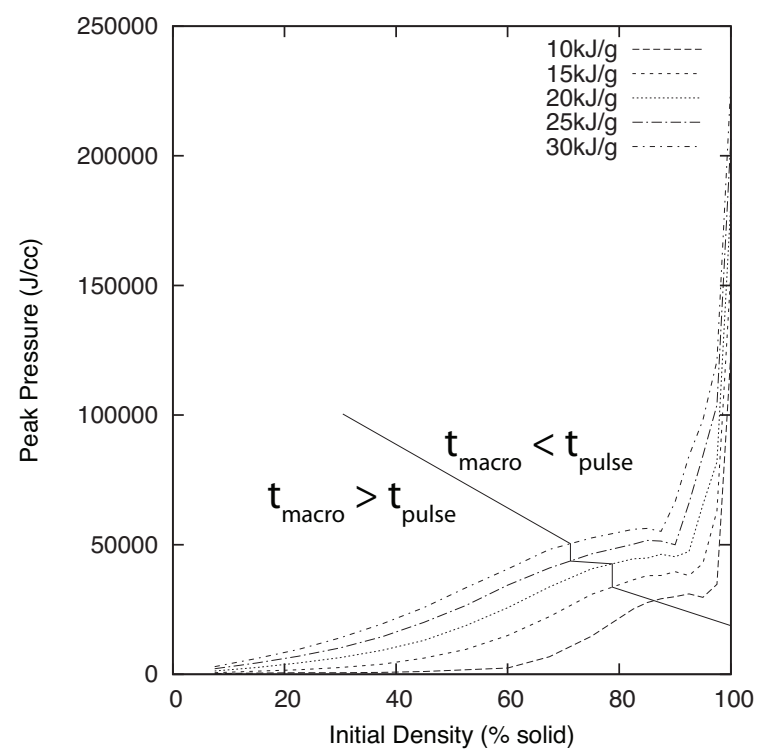

Figure 8: Peak pressure observed vs initial foam density, $0.5 \mathrm{~ns}$ pulses. The solid line connects the points for each energy deposition trend for which $t_{\text {macro }}=$ $t_{\text {pulse. }}$.

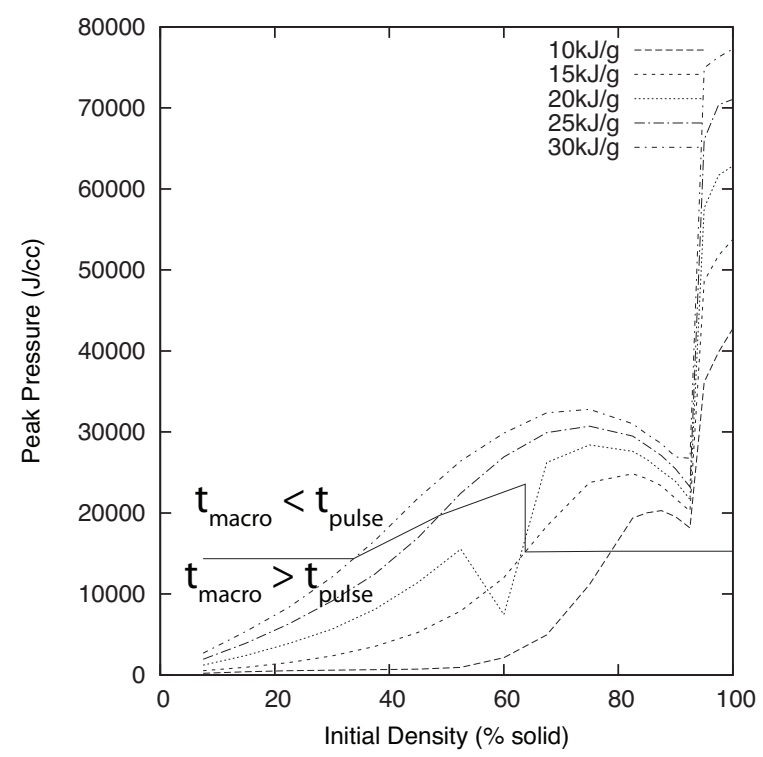

Figure 9: Peak pressure observed vs initial foam density, $1.5 \mathrm{~ns}$ pulses. The solid line connects the points for each energy deposition trend for which $t_{\text {macro }}=$ $t_{\text {pulse. }}$

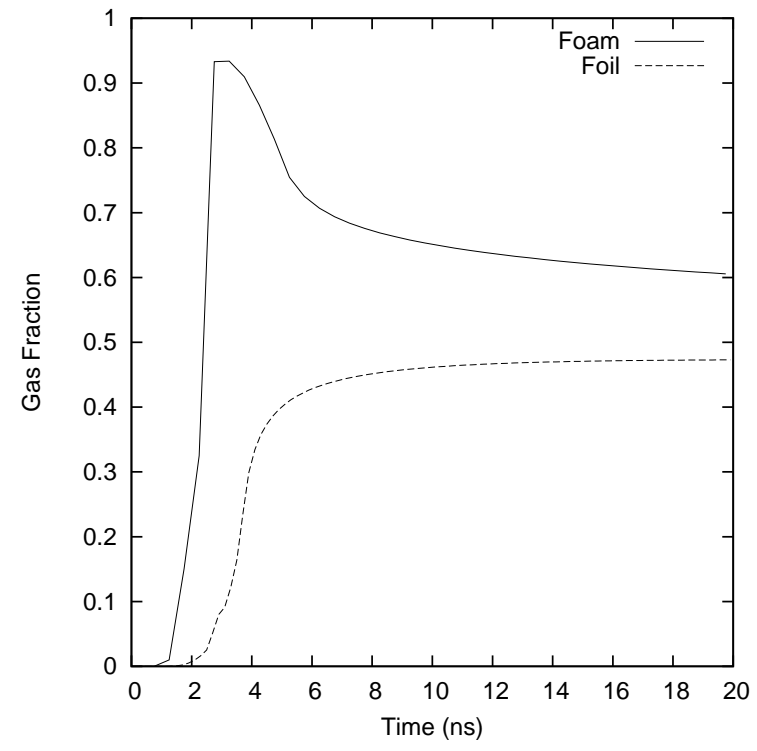

Figure 10: Material gas fraction vs time, 3.0ns pulse with $15 \mathrm{~kJ} / \mathrm{g}$ energy deposition. The foam is $30 \%$ liquid initial density.

\subsection{Phase Evolution}

Another interesting quantity to observe as we compare foams to foils is the phase evolution of the material as it is heated and expands. In particular we calculate the gas fraction for all of the simulated material versus time. In the two-phase region the gas fraction is calculated by extrapolating between the liquid and gas boundaries. Figures 10 and 11 show the gas fraction for both $30 \%$ foams and liquid foils for $3.0 \mathrm{~ns}$ pulses of $15 \mathrm{~kJ} / \mathrm{g}$ and $30 \mathrm{~kJ} / \mathrm{g}$ energy deposition. In the lower energy case, Figure 10 , the foam almost entirely vaporizes during the pulse and then partially condenses, approaching an asymptotic value of the gas fraction between 0.5 and 0.6 . This is because the material is heated above the two phase region and then the material cools along adiabats into the two phase region. We observe several time steps during energy deposition where the material tracks the two-phase boundary from initial cold liquid conditions, until the material is mostly homogenized at around $1 \mathrm{~g} / \mathrm{cc}, 1.4-1.6$ $\mathrm{eV}$ and then cools along adiabats to slighly below the two-phase boundary. The low energy liquid foil, on the other hand, heats and expands through the two phase region, remaining mostly liquid during the pulse and afterwards asymptotically approaching approximately 0.5 gas fraction.

In the higher energy deposition case, Figure 11, we observe both the foam and the foil completely vaporizing during the pulse, and then beginning to condense later as the material cools along adiabats into the two phase region. The foam, however, vaporizes faster than the foil and condenses slower. These two behaviors are qualitatively similar to most of the simulations run. In some cases we also observe neither foam nor foil significantly vaporizing during the pulse, similar to the behavior of the foil in Figure 10.

The cause of differing phase evolution behavior between the foam and foil in these cases results from the initial structure of the foam. As the foam is heated the material quickly expands 


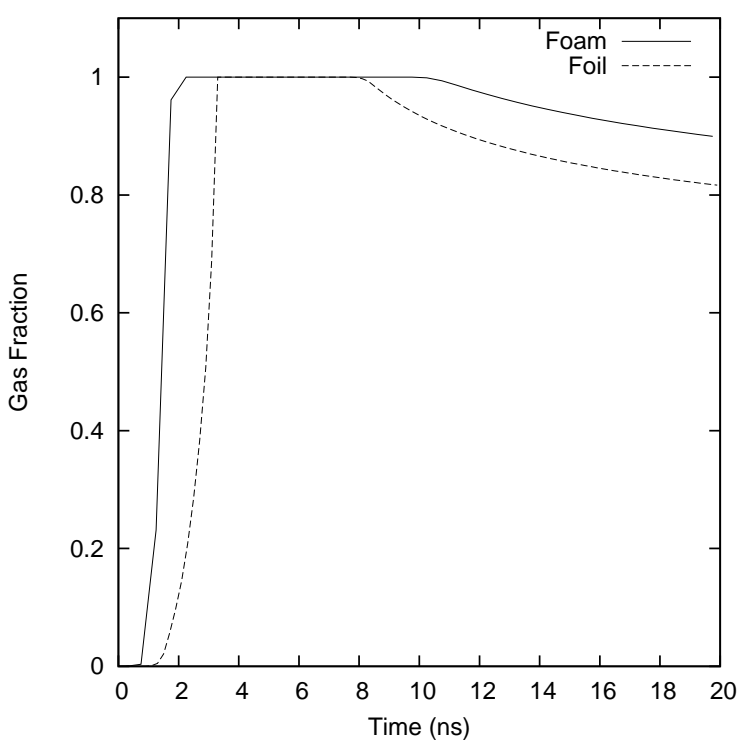

Figure 11: Material gas fraction vs time, 3.0ns pulse with $30 \mathrm{~kJ} / \mathrm{g}$ energy deposition. The foam is $30 \%$ liquid initial density.

into the gap regions. In a $30 \%$ foam this results in a decrease in the density by a factor of 3 . This tends to cause more and rapid vaporization. In the higher energy deposition case, Figure 11, the foam condenses at a slower rate than a foil heated by the same pulse since the hydrodynamic expansion is slower. This suggests that foams more easily achieve complete vaporization: in the low energy case the foam is vaporized while the foil only reaches a gas fraction of $50 \%$. In the higher energy case, where both types of target vaporize, the foam reaches $x_{\text {gas }}=1$ quicker and remains completely vaporized longer than the foil.

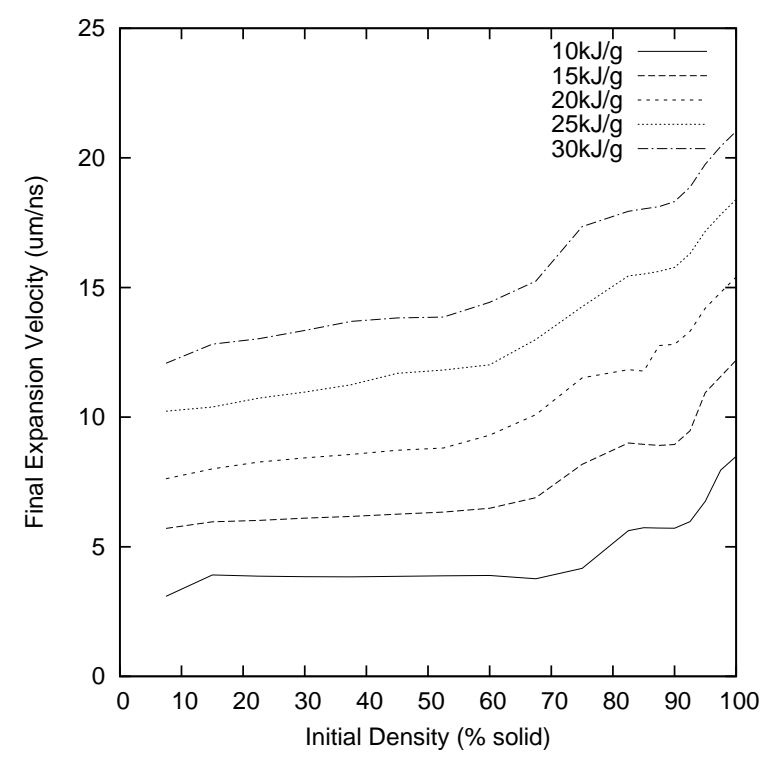

Figure 12: Asymptotic expansion velocity vs initial density, $0.5 \mathrm{~ns}$ pulses of various energy depositions.

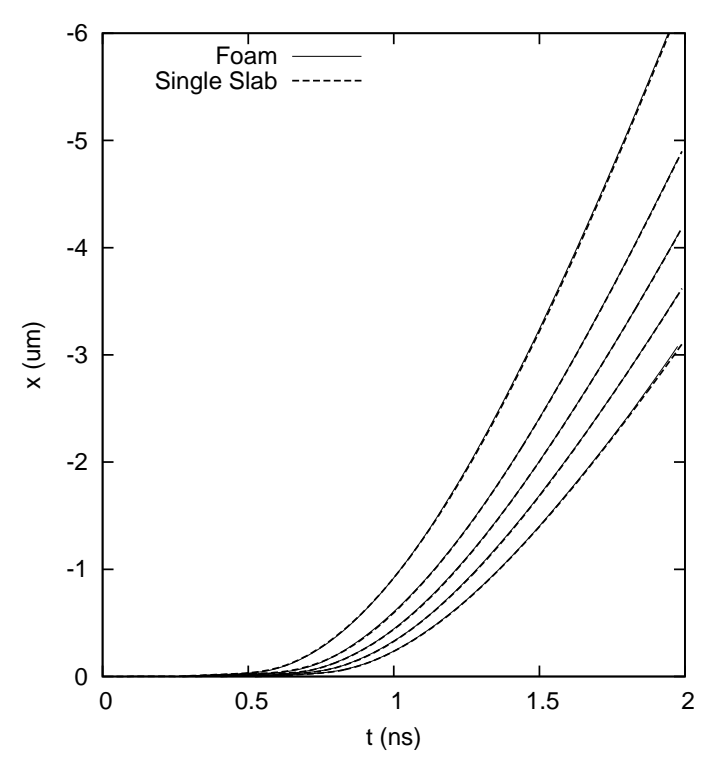

Figure 13: Lagrangian tracks near expansion edge, $45 \%$ foam heated by a $1.5 \mathrm{~ns}$ $15 \mathrm{~kJ} / \mathrm{g}$ pulse. Foam zones are represented by solid lines, while single slab zones are dashed lines. Here we clearly see that the expansion is identical in both cases.

\section{Expansion Velocity}

In Figure 12 the asymptotic expansion velocity at the edge of the simulated material is plotted versus the initial foam density, up to liquid foils. Lagrangian zones where the gradient scale is larger than the zone width are removed from these calculations as numerically unreliable. This qualitative behavior is typical of all pulse lengths. Here the expansion velocity increases very slightly, linearly, up to approximately $60-70 \%$ initial density. This behavior is characteristic of an edge expansion dominated by material from the first slab of material only.

Figure 13 shows the Lagrangian tracks for several zones near the edge of a $45 \%$ foam with a single outer slab simulation overlaid, and we observe that these edge zones track exactly. At higher densities the eventual macro expansion of the foam becomes faster than this initial single wall expansion and begins to increase the overall asymptotic velocity. This is shown in an analogous plot, Figure 14, for a $90 \%$ foam driven by the same pulse parameters compared against a single slab. We can see that the macro expansion dominates the single slab expansion in this case. In Figure 12 this macro expansion determines the asymptotic expansion velocity for foam densities higher than $\sim 60-70 \%$. The upper bound on the expansion velocity for given pulse parameters is given by the case of an instantaneously heated solid foil.

Tauschwitz et. al. observe an enhancement of the expansion velocity due to shocks from slab collisions within the foam propagating to the expansion front [12]. We similarly observe these slab collision shocks. Although the total energy deposition is similar, in their simulations $t_{\text {pulse }}>>t_{\text {homog. This re- }}$ sults in expansion velocities approximately 1 order of magnitude lower than ours. Therefore in our simulations the shocks do not reach the expansion front over time scales of interest. 


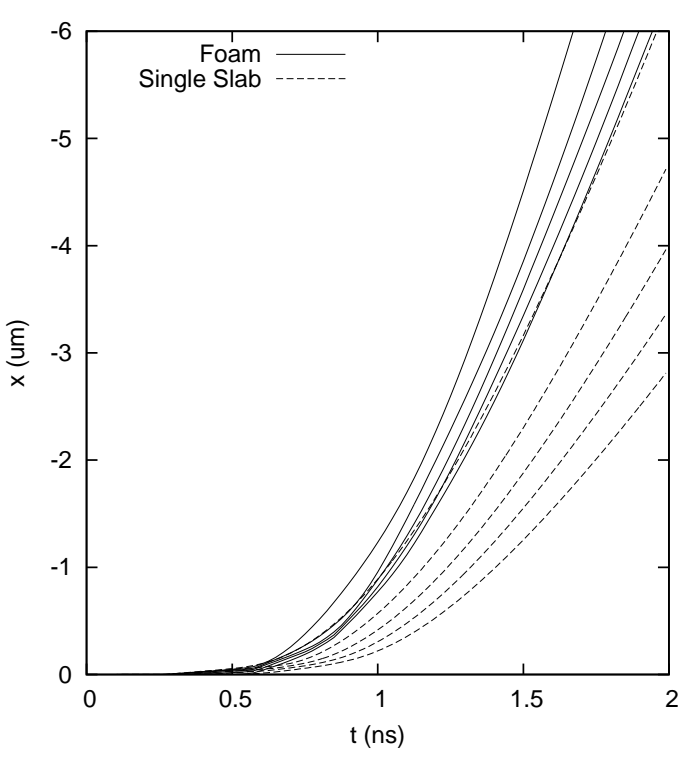

Figure 14: Lagrangian tracks near expansion edge, 90\% foam heated by a $1.5 \mathrm{~ns} 15 \mathrm{~kJ} / \mathrm{g}$ pulse. Foam zones are represented by solid lines, while single slab zones are dashed lines. Early in the expansion, between 0.5 and $1.0 \mathrm{~ns}$, the macroscopic expansion causes the foam zones to accelerate outwards at a greater rate than the single slab zones.

Additionally, the randomized nature of our foams reduce the periodicity between successive shocks.

\section{Foam Homogenization}

In Section 2 we described methods for calculating the inhomogeneity in a foam target, which is calculated as

$$
H=\sqrt{\frac{1}{n} \sum_{i=1}^{n}\left(\frac{\rho_{a}-\rho_{i}}{\rho_{a}}\right)^{2}},
$$

where $n$ is the number of central zones that we use and $\rho_{a}$ is a volume weighted average density over those zones. The inhomogeneities are driven by the initial density distribution, as the foam has constant initial temperature and the energy deposition is volumetric.

This level of inhomogeneity is plotted versus time, as an example, for five $30 \%$ foams heated by $0.5 \mathrm{~ns}$ pulses of various energy deposition in Figure 15. The foams initially start out with a high level of inhomogeneity, as we would expect, and rapidly homogenize to a final 'plateau', representing some amount of persistent inhomogeneity characteristic of foams after homogenization in our simulations. In Figure 15 we can also see that foams heated by higher levels of energy deposition homogenize faster, as expected. For a very low beam intensity $(10 \mathrm{~kJ} / \mathrm{g})$ the foam takes a very long time to homogenize.

We also compute a final level of homogenization, which is defined as the level of inhomogeneity immediately after the defined homogenization time. This is plotted versus the initial foam density for various energy depositions of $0.5 \mathrm{~ns}$ pulses in Figure 16. A trend towards more homogenization in higher density foams is observed.

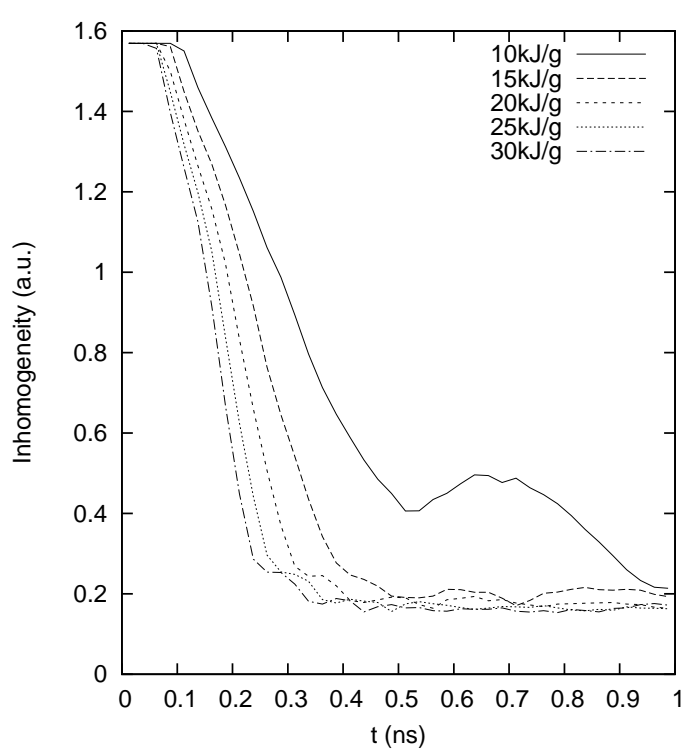

Figure 15: Inhomogeneity versus time, $30 \%$ foams heated by $0.5 \mathrm{~ns}$ pulses of various energy depositions.

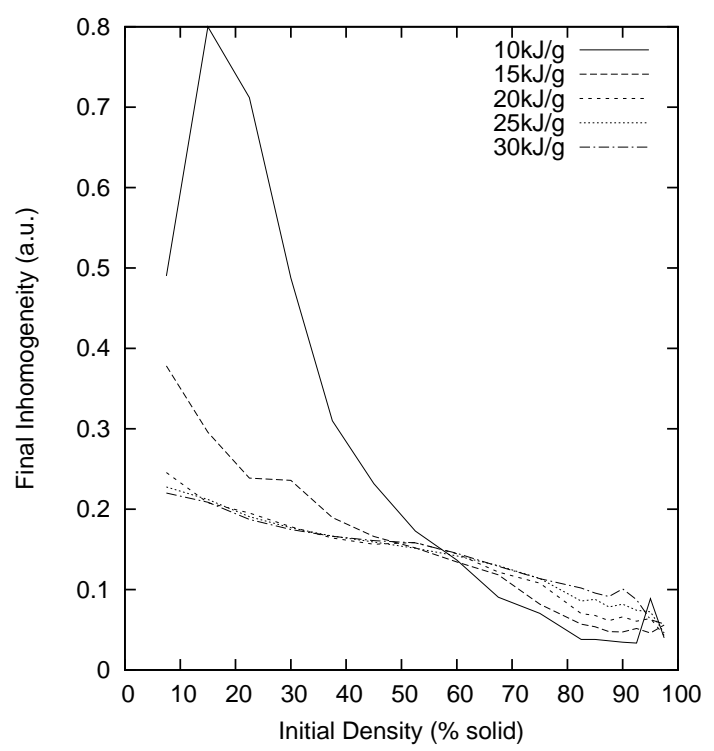

Figure 16: Final level of inhomogeneity versus initial foam density, $0.5 \mathrm{~ns}$ pulses. 
We also examine how the homogenization time depends on the initial density, which is plotted in Figure 17. Shorter homogenization times are observed for higher density foams. The process of foam homogenization can be thought of as a series of rarefaction and compression waves propagating between adjacent liquid and gap regions, asymptotically approaching the final level of inhomogeneity with each iteration of the rarefaction/compression process. In this model the speed at which homogenization will occur depends on the sound speed: therefore, higher sound speeds in higher density foams (see Figure 4) cause lower homogenization times in those foams.

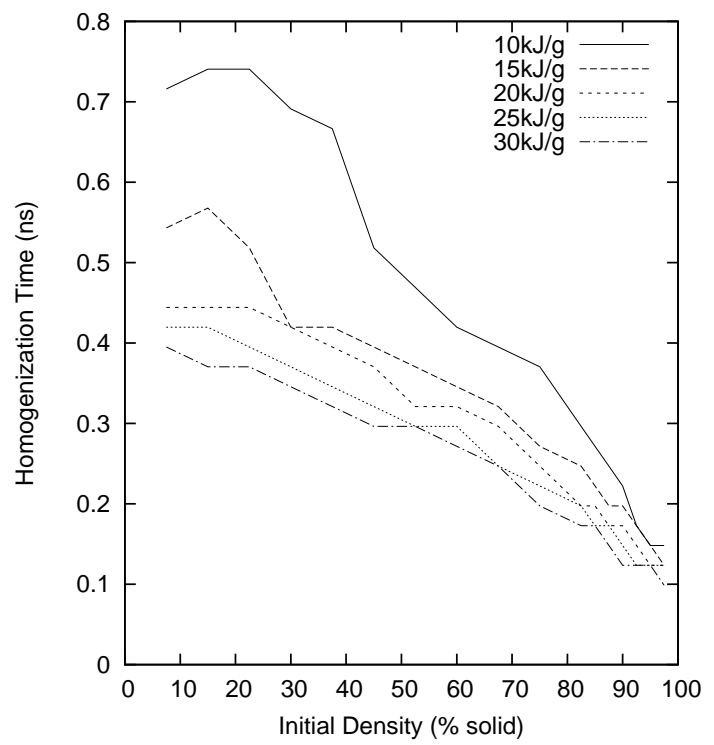

Figure 17: Homogenization time versus initial density, $0.5 \mathrm{~ns}$ pulses.

Finally, we can examine the relation between the pulse length and homogenization time, which is plotted in Figure 18. In this parameter regime the homogenization time is similar to the pulse length and roughly linearly dependent on the pulse length.

The absolute homogenization time will depend on the foam modeling, specifically on the number of slabs. The order of the homogenization time can be estimated by realizing that an idealized homogenization process consists of the hydrodynamic motion of the slabs into the gaps, or the collision time scale.

\section{Conclusions}

A study of various behaviors of foams heated by ion beam energy deposition has been conducted, along with the development of a characterization of time scales in the problem. We find that, as the foam density is varied, the peak temperature reached in a foam is highest for foam densities where the macroscopic rarefaction wave reaches the center of the foam at the end of the pulse length. At fixed $t_{\text {pulse }}$ and $E_{\text {dep }}$ with $\rho_{\text {foam }}$ such that $t_{\text {macro }}>t_{\text {pulse }}$ decreasing the foam density further decreases the temperature due to a cooling effect when the foam slabs expand into the gaps, which is greater for lower densities. Likewise, with fixed $t_{\text {pulse }}$ and $E_{\text {dep }}$ and $\rho_{\text {foam }}$ such that

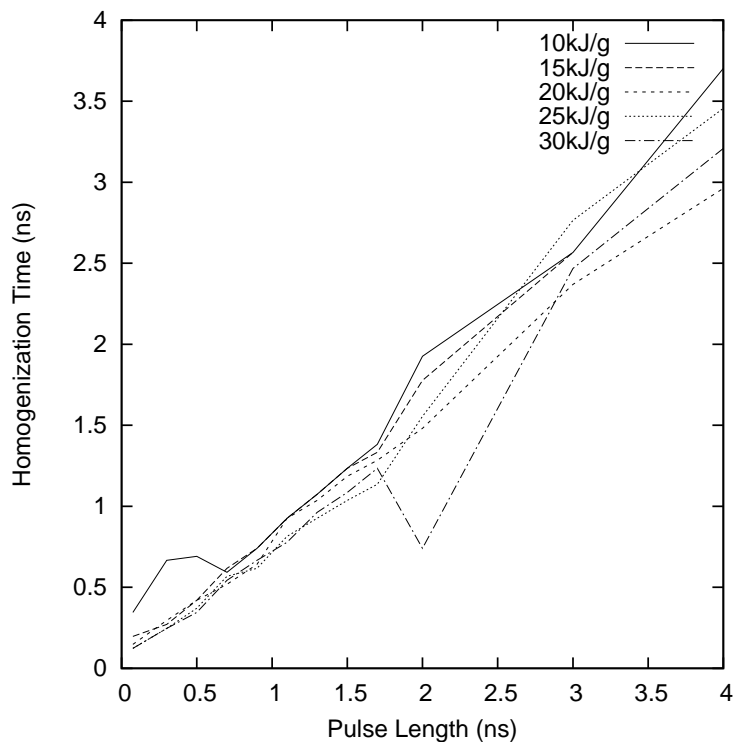

Figure 18: Homogenization time versus pulse length, 30\% foams.

$t_{\text {macro }}<t_{\text {pulse }}$ the macroscopic expansion cools the foam center before the energy deposition is complete, resulting in lower peak temperatures. Additionally, as expected, peak foam temperatures increase with increasing energy deposition at fixed $\rho_{\text {foam }}, t_{\text {pulse }}$.

Studies of the peak pressure imply increased pressures for higher energy depositions and shorter pulses. In very high density foams we observe peak pressures before homogenization, since the near liquid density slabs are in a phase regime where the Van der Waals equation of state gives unphysical pressures. In the lower density foams a maximum is observed in the peak pressure versus density. Because of the Van der Waals pressure density dependence this occurs at a higher foam density than the peak temperature does.

For fixed $t_{\text {pulse }}, E_{\text {dep }}$ the expansion velocity is found to be roughly constant for foam densities below a certain critical density. Below this density the single slab expansion on the edge of the foam dominates the overall expansion. For higher densities the bulk expansion dominates, resulting in higher velocities.

When studying the phase evolution we find that at some NDCX-II relevant parameters foam targets vaporize completely while solid targets do not at the same pulse parameters. Both targets are observed to trend towards the same asymptotic gas fraction. This suggests that foam targets are more easily completely vaporized for fixed $t_{\text {pulse }}, E_{\text {dep }}$.

We developed a method for evaluating the homogenization of foams versus time, including the time it takes foams to homogenize and levels of persistent inhomogeneity in the foams. We find that this level of persistent inhomogeneity and the time for homogenization both decrease with increasing foam density. The time for homogenization is roughly proportional to the pulse length.

These studies focus on the idealized dynamics of foam targets, and thus have been conducted in 1-D with approximations such as uniform heating and a single total thickness of the foam. 
In a real foam, phenomena such as gap collapses are highly dependent on dimensionality and may result in deviations from the one dimensional case. It is our expectation, however, that the qualitative behavior and dynamics of the real foams when parameterized by the appropriate analogous three dimensional time scales will show similarities to the behaviors found in 1D foams. Additionally, the Van der Waals with Maxwell construction does not include information on the dynamics of droplet formation. This theoretical and computational study of fundamental foam dynamics will be applied to upcoming experiments at LBNL and further theory work, including higher dimension simulations and more detailed equations of state.

\section{Acknowledgments}

We would like to thank F. Bieniosek and P. Ni for useful discussions regarding foam targets and relevant experiments. This work was performed under the auspices of the U.S. Department of Energy under contract DE-AC52-07NA27344 at LLNL, and University of California contract DE-AC02-05CH11231 at LBNL.

\section{References}

[1] Barnard, J., Armijo, J., More, R., Friedman, A., Kaganovich, I., Logan, B., Marinak, M., Penn, G., Sefkow, A., Santhanam, P., et al., 2007. Theory and simulation of warm dense matter targets. Nuclear Inst. and Methods in Physics Research, A 577 (1-2), 275-283.

[2] Becker, R., Leibfried, G., 1967. Theory of heat. Springer.

[3] Bieniosek, F., Henestroza, E., Leitner, M., Logan, B., More, R., Roy, P., Ni, P., Seidl, P., Waldron, W., Barnard, J., 2009. High-energy density physics experiments with intense heavy ion beams. Nuclear Inst. and Methods in Physics Research, A.

[4] Efremov, V., Demidov, B., Mescheryakov, A., Potapenko, A., Fortov, V., 2007. Physical modeling of porous media behavior in targets for inertial fusion. Nuclear Inst. and Methods in Physics Research, A 577 (1-2), 324 326.

[5] Friedman, A., Barnard, J., Briggs, R., Davidson, R., Dorf, M., Grote, D., Henestroza, E., Lee, E., Leitner, M., Logan, B., et al., 2009. Toward a physics design for NDCX-II, an ion accelerator for warm dense matter and HIF target physics studies. Nuclear Inst. and Methods in Physics Research, A.

[6] Milovich, J., Amendt, P., Hamza, A., Marinak, M., Robey, H., 2006. Highly-resolved 2D HYDRA simulations of Double-Shell Ignition Designs. UCRL-PROC-222562, Lawrence Livermore National Laboratory (LLNL), Livermore, CA.

[7] More, R. M., 2008. DISH User Manual. LBNL Report.

[8] Roy, P., Seidl, P., Anders, A., Bieniosek, F., Coleman, J., Gilson, E., Greenway, W., Grote, D., Jung, J., Leitner, M., et al., 2009. A spacecharge-neutralizing plasma for beam drift compression. Nuclear Inst. and Methods in Physics Research, A.

[9] Seidl, P., Anders, A., Bieniosek, F., Barnard, J., Calanog, J., Chen, A., Cohen, R., Coleman, J., Dorf, M., Gilson, E., et al., 2009. Progress in beam focusing and compression for warm-dense matter experiments. $\mathrm{Nu}$ clear Inst. and Methods in Physics Research, A.

[10] Tahir, N., Shutov, A., Lomonosov, I., Piriz, A., Wouchuk, G., Deutsch, C., Hoffmann, D., Fortov, V., 2006. Numerical simulations and theoretical analysis of high energy density experiments at the next generation of ion beam facilities at darmstadt: The hedgehob collaboration. High Energy Density Physics 2 (1-2), $21-34$.

[11] Tahir, N., Spiller, P., Udrea, S., Cortazar, O., Deutsch, C., Fortov, V., Gryaznov, V., Hoffmann, D., Lomonosov, I., Ni, P., et al., 2006. Studies of equation of state properties of high-energy density matter using intense heavy ion beams at the future FAIR facility: The HEDgeHOB collaboration. Nuclear Inst. and Methods in Physics Research, B 245 (1), 85-93.
[12] Tauschwitz, A., Efremov, V., Maruhn, J., Rosmej, F., Tauschwitz, A., 2009. Quasi-static heating of stack targets with intense ion beams for equation of state measurements. Nuclear Inst. and Methods in Physics Research, B. 